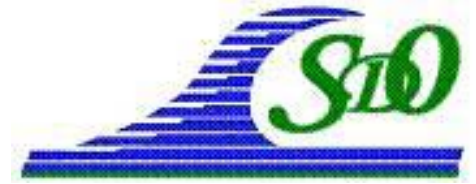

XI ${ }^{\text {èmes }}$ Journées Nationales Génie Côtier - Génie Civil

Les Sables d'Olonne, 22-25 juin 2010

DOI:10.5150/jngcgc.2010.031-G @ Editions Paralia CFL

disponible en ligne - http://www.paralia.fr - available online

\title{
Dynamique sédimentaire d'un estran lagunaire colonisé par un herbier de zostères (Bassin d'Arcachon, France)
}

\author{
Florian GANTHY $^{1,2}$, Aldo SOTTOLICHIO ${ }^{1}$, Romaric VERNEY ${ }^{2}$
}

1. Université Bordeaux 1, Laboratoire EPOC UMR 5805 CNRS, Avenue des Facultés, 33405 Talence cedex, France.

f.ganthy@epoc.u-bordeauxl.fr; a.sottolichio@epoc.u-bordeauxl.fr

2. IFREMER, Département Dynamiques de l'Environnement Côtier, équipe PHYSED, BP70, 29280 Plouzané, France.

Romaric.Verney@ifremer.fr

\section{Résumé :}

Le bassin d'Arcachon, lagune côtière semi-fermée, possède de vastes herbiers de phanérogames (Zostera noltii et Z. marina). Ces dernières années, la surface occupée par les herbiers s'est sensiblement réduite. L'un des enjeux majeurs actuels du bassin d'Arcachon réside dans l'évaluation des conséquences de cette régression sur la dynamique sédimentaire de la lagune. Cependant, la connaissance des interactions entre les processus hydro-sédimentaires et les évolutions saisonnières des herbiers de zostères reste faible. Dans ce contexte, l'étude conjointe et à différentes échelles de temps des caractéristiques hydrodynamiques et sédimentaires des estrans et de la biométrie des zostères, permet de mieux comprendre les processus sédimentaires en présence d'herbiers.

Dans cet objectif, un estran atelier présentant des densités d'herbiers variables a été instrumenté et étudié sur un cycle annuel. La stratégie de mesure a consisté à mesurer à haute fréquence, sur plusieurs stations (allant d'un herbier dense à un estran nu), les principaux paramètres hydro-sédimentaires (altimétrie du sédiment, hauteurs de marée, vagues) conjointement à la dynamique de l'herbier (suivi biométrique mensuel). Les premiers résultats, portant sur la période de croissance des zostères et le début de la période de dégénérescence automnale, mettent en évidence une accrétion sédimentaire significative de l'estran, positivement corrélée au développement de l'herbier, ainsi qu'une protection de l'estran contre l'érosion lorsque l'herbier est développé. La poursuite du suivi en période hivernale devrait permettre d'observer les tendances lors de l'absence hivernale de l'herbier et de déterminer le bilan sédimentaire annuel de l'estran.

\section{Mots-clés :}

Dynamique sédimentaire - Herbiers de zostères - Evolutions saisonnières - Altimétrie des sédiments - Mesures biométriques 


\section{Introduction}

Les herbiers situés en zones côtières peu profondes sont connus pour (i) atténuer l'énergie hydrodynamique issue des courants et des vagues (FONSECA \& FISHER, 1986 ; ABDELRHMAN, 2003 ; MOLLER, 2006), (ii) favoriser le dépôt sédimentaire (GACIA et al., 2003) et (iii) protéger le substrat contre l'érosion (AMOS et al., 2004 ; THOMPSON et al., 2004 ; BOS et al., 2007). Cependant, la majorité des études dédiées au rôle de la végétation sur les processus sédimentaires ont été menées sur des espèces subtidales pérennes (Posidonia oceanica, GACIA et al., 2003), ou sur des espèces colonisant les schorres (Spartina anglica, MOLLER, 2006). Aussi, la connaissance des interactions entre les processus hydro-sédimentaires et les évolutions saisonnières d'herbiers intertidaux constitués d'espèces annuelles, telles que Zostera noltii, reste faible (BOS et al., 2007). Par ailleurs, dans de nombreuses lagunes européennes, une diminution de la surface occupée par des herbiers marins intertidaux a été mise en évidence au cours du siècle dernier (BOS et al., 2007 ; DE BOER, 2007). S'il semble établi que les causes de ces régressions des surfaces sont des perturbations anthropiques, les conséquences de celles-ci restent encore mal appréhendées.

La lagune côtière d'Arcachon (voir figure 1), située sur le littoral aquitain, possède de vastes herbiers de phanérogames (Zostera noltii) dont la surface occupée s'est réduite d'environ 33\% entre 1989 et 2007 (DALLOYAU et al., 2009). L'un des enjeux majeurs actuels du bassin d'Arcachon réside dans l'évaluation des conséquences de cette régression sur les bilans sédimentaires de la lagune. En effet, en parallèle de cette régression, une tendance au comblement des chenaux d'accès aux ports de fond de bassin a été observée sur la même période, laissant supposer une relation entre ces deux phénomènes. Pour cette raison, un travail expérimental a été entrepris dans le but de comprendre comment les évolutions saisonnières des herbiers interviennent dans la modification des processus d'érosion/dépôt des sédiments.

Dans le présent article, les premiers résultats de ce suivi expérimental sont présentés et discutés, en visant deux objectifs : (i) une évaluation préalable de la méthodologie de suivi adoptée, basée sur l'altimétrie acoustique, et (ii) une première analyse des conséquences de la dynamique saisonnière des herbiers intertidaux de Zostera noltii sur la dynamique sédimentaire.

\section{Matériel et méthodes}

Un estran atelier présentant un herbier stable dans le temps a été choisi pour faire l'objet d'un suivi simultané des caractéristiques biométriques et des évolutions hydrosédimentaires. Quatre stations y ont été mises en place: herbier dense, herbier moyennement dense, herbier peu dense et une station témoin d'estran nu. Leur proximité (moins de $100 \mathrm{~m}$ entre 2 stations consécutives) permet de faire l'hypothèse que les conditions hydrodynamiques y sont similaires. La méthodologie du suivi consiste en un enregistrement haute fréquence des paramètres hydro-sédimentaires et en 


\section{XI ìmes Journées Nationales Génie Côtier-Génie Civil}

Les Sables d'Olonne, 22-25 juin 2010

une mesure mensuelle des caractéristiques de l'herbier. Le suivi a démarré le 11 février 2009 (il se poursuivra jusqu'en février 2010) et les résultats présentés ici couvrent la période allant jusqu'au 5 décembre 2009.

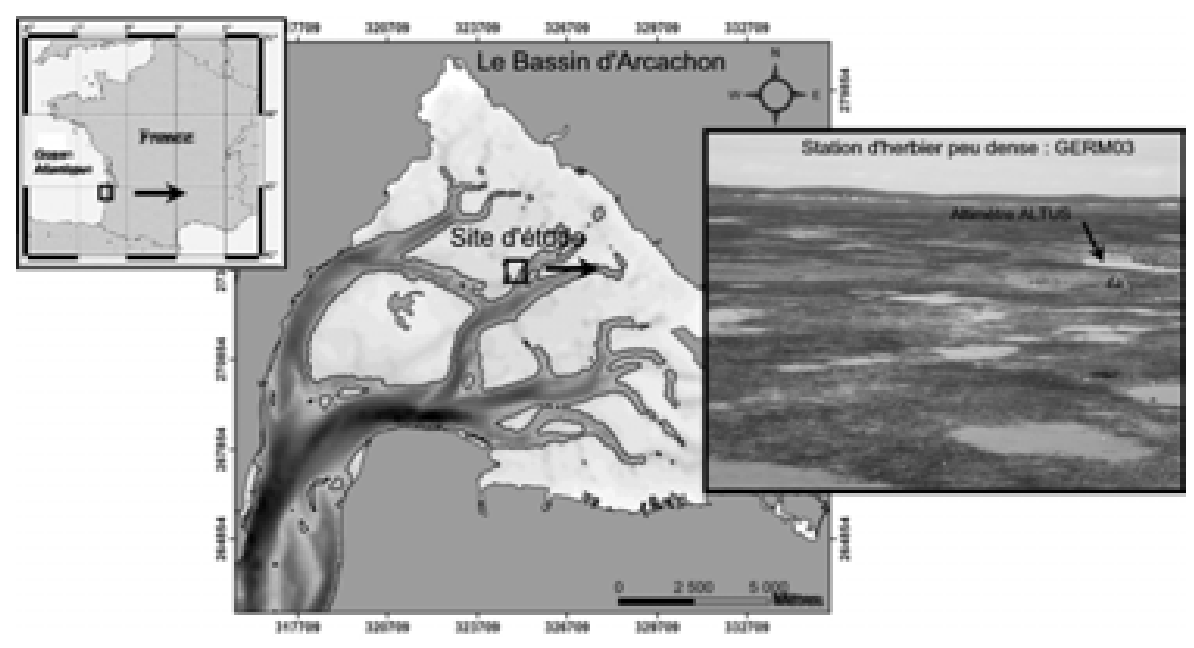

Figure 1. Localisation de la zone d'étude.

\subsection{Biométrie des zostères}

Le suivi biométrique réalisé mensuellement dans cette étude se base sur le protocole de la Directive Cadre sur l'Eau (D.C.E). Neuf échantillons sont prélevés par station au moyen de micro-carottes de $98 \mathrm{~mm}$ de diamètre et de $70 \mathrm{~mm}$ de profondeur. Les échantillons sont lavés à l'eau douce sur un tamis afin de les séparer du sédiment. L'analyse biométrique consiste à séparer les pousses du système racinaire, et à mesurer par analyse d'image la longueur et la largeur de toutes les feuilles de 10 pousses représentatives de l'échantillon. Le nombre total de pousses est ensuite compté. Pour chaque échantillon sont calculés la longueur moyenne des feuilles et l'indice de surface foliaire (I.S.F., surface de feuille par unité de surface de substrat). Pour chaque station (9 échantillons), la moyenne et l'écart-type de chaque paramètre sont calculés.

\subsection{Altimétrie du sédiment}

Des mesures d'altimétrie du sédiment ont été réalisées en continu au moyen d'ALTUS (JESTIN et al., 1998; BASSOULLET et al., 2000). Ces appareils autonomes fonctionnent sur le principe d'un échosondeur. Le transducteur émet une onde acoustique de fréquence $2 \mathrm{MHz}$ et joue le rôle d'émetteur et de récepteur. Les Altus sont déployés sur un trépied, afin de limiter au maximum l'affouillement sous le transducteur. La précision de la mesure altimétrique est de $\pm 2 \mathrm{~mm}$ pour une distance entre le transducteur et le substrat comprise entre 0.2 et $0.7 \mathrm{~m}$, tandis que la résolution est de $0.6 \mathrm{~mm}$. Ces appareils permettent d'enregistrer jusqu'à quatre mesures d'altimétrie simultanées, dont chacune est basée sur une valeur seuil de l'intensité du signal de retour. Dans cette étude, les valeurs ont été fixées, respectivement à 75, 35, 17 
et $10 \%$ du signal maximum. La fréquence d'échantillonnage est définie à $2 \mathrm{~Hz}$ par salves de 2 minutes 30 secondes, espacées de 10 minutes.

Le traitement des données à été réalisé sous MATLAB ${ }^{\circledR}$. Une chaine de post-traitement spécifique a été développée et appliquée pour analyser la capacité de l'ALTUS à mesurer l'évolution du fond sédimentaire en présence d'herbiers. Pour chaque salve de mesure, et pour chaque seuil, les distances maximales, moyennes et minimales ont été calculées. Lors de certaines visites mensuelles, les zostères ont été coupées sous les capteurs, de manière à maintenir dégagé le volume insonifié et à empêcher le recouvrement du transducteur par les feuilles. Enfin, lors de chaque visite du site, la distance entre le sédiment et le transducteur est mesurée manuellement afin de vérifier la fiabilité des données acquises.

\section{Résultats}

\subsection{Capacité de mesure du niveau sédimentaire en présence de végétation}

Avant cette étude, l'ALTUS a été principalement utilisé sur estrans sablo-vaseux, exempts de végétation (BASSOULLET et al., 2000 ; DELOFFRE et al., 2007). La présence d'herbier peut potentiellement influencer la réponse acoustique. Il est donc nécessaire d'examiner la capacité de l'ALTUS à mesurer la distance transducteur/fond dans cet environnement spécifique.

Sur un estran nu, le sédiment constitue un réflecteur fort et la signature du signal acoustique se caractérise par une forte pente : le franchissement des seuils se produit à des intervalles de temps rapprochés, correspondant à des altimétries similaires (voir figure 2, cas A).

En présence d'herbiers, les feuilles de zostères se comportent également comme des réflecteurs: le signal acoustique réfléchi se traduit alors schématiquement par une double signature, la première due aux feuilles, la seconde due au sédiment (voir figure 2, cas B). Dans certains cas, le signal réfléchi attribué aux feuilles peut être fort, jusqu'à empêcher la détection du pic généré par le sédiment (voir figure 2, cas C). On fait ici l'hypothèse suivante: les distances maximales entre le point de mesure et le transducteur, calculées par salve pour le seuil 1 (75\%) correspondent au substrat sédimentaire, tandis que les distances minimales calculées pour le seuil 4 (10\%) sont associées au sommet de la canopée de zostères. Une première investigation a été menée afin de vérifier cette hypothèse, ainsi que la validité des données altimétriques en présence de zostères.

Dans le tableau 1 sont présentées, lors des coupes et sous différentes formes, les distances sédiment-transducteur des deux sites GERM01 et GERM04: HM est la mesure manuelle effectuée à marée basse. Les mesures ALTUS du seuil 1 (H1moy, H1std, H1max), sont respectivement la moyenne, l'écart-type et le maximum de la dernière salve avant, et de la première salve après la mesure manuelle HM. 


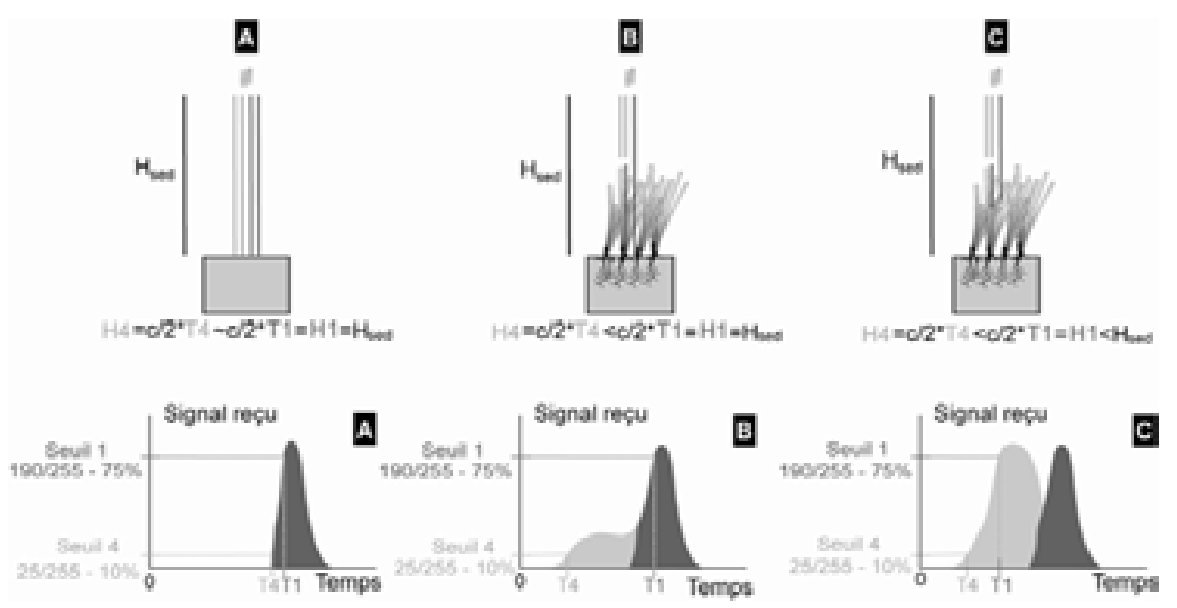

Figure 2. Schéma explicatif des différents seuils de mesure des ALTUS, en l'absence (cas $A$ ) et en présence (cas B et $C$ ) de zostères sous le capteur.

Tableau 1. Synthèse des relevés d'altimétrie du sédiment (distance verticale sondesubstrat) pour les stations d'herbier dense (GERM01) et d'estran nu (GERM04), lors des coupes de zostères. Mesure manuelle (HM) effectuée à marée basse et enregistrements ALTUS du seuil 1 avant et après la mesure HM (H1moy: moyenne de la salve ; H1std : écart-type ; H1max : maximum de la salve).

\begin{tabular}{|c|c|c|c|c|c|c|c|c|c|c|}
\hline \multirow[b]{3}{*}{ Dates } & \multicolumn{5}{|c|}{ GERM01 } & \multicolumn{5}{|c|}{ GERM04 } \\
\hline & \multicolumn{2}{|c|}{ Avant $\mathrm{HM}$} & \multirow[b]{2}{*}{$\begin{array}{c}\mathrm{HM} \\
{[\mathrm{mm}]}\end{array}$} & \multicolumn{2}{|c|}{ Après HM } & \multicolumn{2}{|c|}{ Avant HM } & \multirow[b]{2}{*}{$\begin{array}{c}\mathrm{HM} \\
{[\mathrm{mm}]}\end{array}$} & \multicolumn{2}{|c|}{ Après HM } \\
\hline & $\begin{array}{c}\text { H1 moy } \pm \\
\text { H1 std [mm] }\end{array}$ & $\begin{array}{l}\mathrm{H} 1 \mathrm{max} \\
{[\mathrm{mm}]}\end{array}$ & & $\begin{array}{c}\text { H1moy } \pm \\
\text { H1std [mm] }\end{array}$ & $\begin{array}{c}\text { H1 max } \\
{[\mathrm{mm}]}\end{array}$ & $\begin{array}{c}\text { H1moy } \pm \\
\text { H1std [mm] }\end{array}$ & $\begin{array}{l}\mathrm{H} 1 \mathrm{max} \\
{[\mathrm{mm}]}\end{array}$ & & $\begin{array}{c}\text { H1moy } \pm \\
\text { H1std [mm] }\end{array}$ & $\begin{array}{c}\mathrm{H} 1 \mathrm{max} \\
{[\mathrm{mm}]}\end{array}$ \\
\hline $11 / 02$ & 10.0. & N.D. ${ }^{*}$ & $327^{*}$ & $325.8 \pm 0.0^{*}$ & . & (1) & . & 28 & & 282.1 \\
\hline $08 / 04$ & $300.1 \pm 4.8^{*}$ & $314.3^{*}$ & $315^{*}$ & $310.9 \pm 1.7^{*}$ & $310.9^{*}$ & $272.6 \pm 0.8$ & 273.8 & 274 & $270.7 \pm 1.3$ & 270.7 \\
\hline $28 / 05$ & $279.4 \pm 16.3^{*}$ & $294.3^{*}$ & $295^{*}$ & $290.9 \pm 2.5^{*}$ & $294.8^{*}$ & $279.5 \pm 0.2$ & 279.7 & 280 & $280.6 \pm 0.2$ & 280.9 \\
\hline
\end{tabular}

On y remarque que H1moy diffère sensiblement de HM, alors que les H1max avant et après HM y sont sensiblement égales. Les écart-types sont bien plus importants avant HM qu'après HM. Ensuite, sur la figure 3, qui représente pour les différentes stations, les distances maximales entre le point de mesure et le transducteur, calculées par salve pour le seuil 1 (75\%, voir figure 3 , points noirs), on peut remarquer une répartition étroite du nuage de points. Cette distribution définit une interface distincte, corrélée aux mesures manuelles réalisées mensuellement entre le substrat sédimentaire et le transducteur (voir tableau 1 et figure 3, triangles noirs). La mesure donnée par le maximum du seuil 1 correspond donc bien au substrat sédimentaire, confirmant la validité des données ALTUS en présence d'herbiers. 


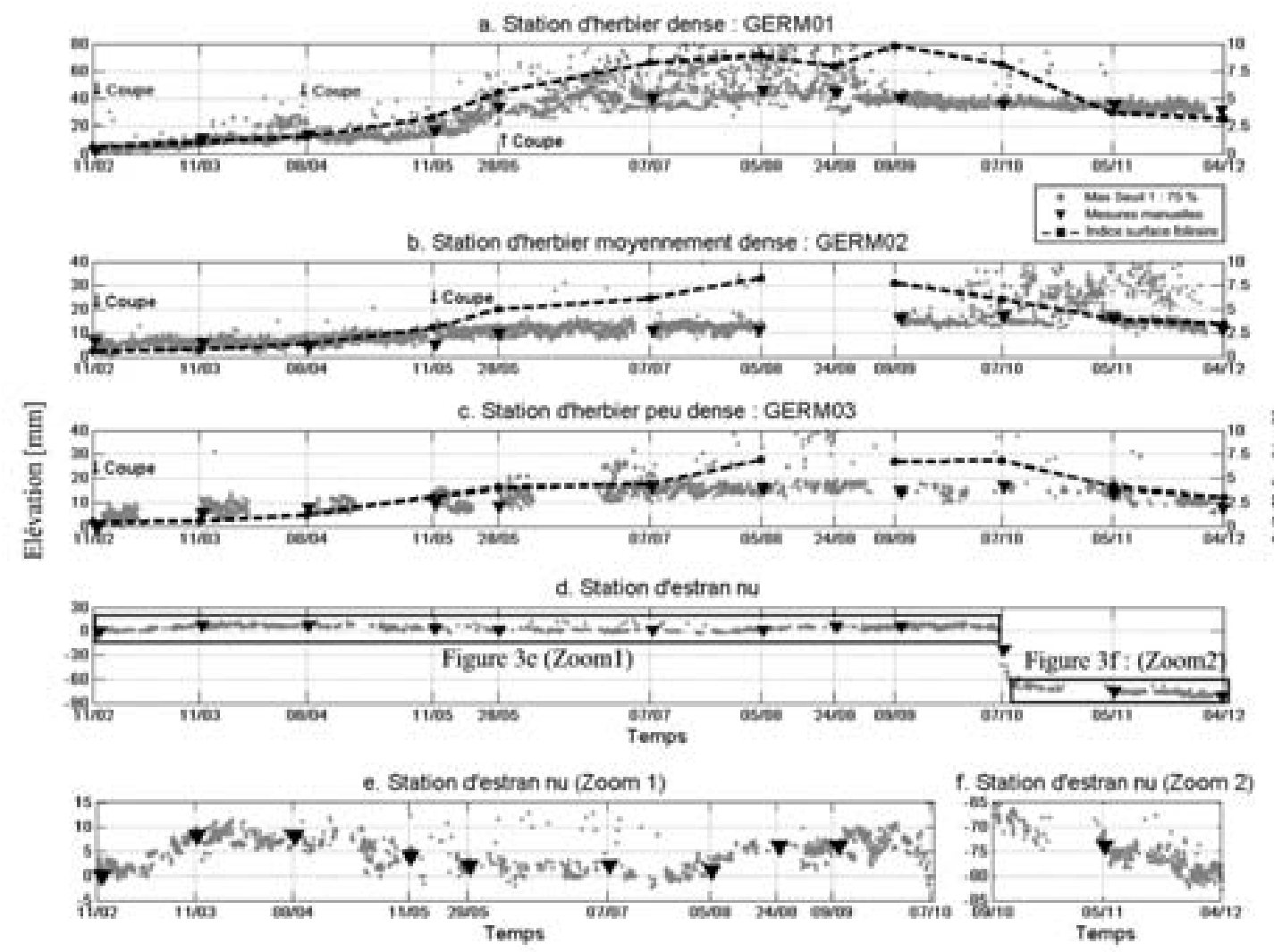

Figure 3. Mesures ALTUS (seuil 1 - points gris) et données d'Indice de Surface Foliaire (I .S.F. - trait pointillé noir) calculées à partir des prélèvements biométriques. Les quatre stations ont été représentées : (a) l'herbier dense, (b) l'herbier moyennement dense, (c) l'herbier peu dense et (d, e et f) l'estran nu.

\subsection{Evolution saisonnière du substrat sédimentaire en présence d'herbier}

Afin d'étudier l'évolution saisonnière du niveau sédimentaire, les données altimétriques du seuil 1, correspondant au niveau sédimentaire, sont représentées dans la figure 3 (points gris) et comparées aux mesures manuelles (triangles noirs) et à l'indice de surface foliaire calculé à partir des prélèvements biométriques mensuels (pointillés et carrés noirs). Une élévation continue du substrat est observée sur la période de février à octobre, pour les trois stations colonisées par les zostères (voir figures $3 \mathrm{a}$ à $3 \mathrm{c}$ ). L'accrétion sédimentaire s'avère être maximum pour la station située dans l'herbier dense (40 mm, voir figure 3a). L'accrétion est plus faible pour les stations d'herbier moyennement dense et peu dense (respectivement $13 \mathrm{~mm}$ et $15 \mathrm{~mm}$, voir figure $3 \mathrm{~b}$ et 3c). L'indice de surface foliaire augmente sur la même période, traduisant quantitativement la pousse saisonnière de l'herbier. On observe ensuite, à partir du mois d'octobre, une diminution lente et de faible amplitude du niveau sédimentaire pour les trois stations d'herbier $(-15 \mathrm{~mm},-7 \mathrm{~mm}$ et $-8 \mathrm{~mm}$, respectivement pour les stations d'herbier dense, moyennement dense et peu dense). 
En revanche, pour la station située sur l'estran nu (voir figure 3d à 3f) on observe, sur la période du 11 février au 7 octobre, une alternance de phases d'augmentation et de diminution du niveau sédimentaire d'amplitude inférieure à $10 \mathrm{~mm}$. Entre le 7 octobre et le 9 octobre, une diminution importante $(-60 \mathrm{~mm})$ intervient, puis la diminution se poursuit ensuite plus lentement $(-15 \mathrm{~mm}$ entre le 9 octobre et le 4 décembre). On observe donc des dynamiques différentes entre les stations colonisées et la station d'estran nu.

\section{Discussion}

\subsection{Estimation de la longueur des feuilles de zostères par les ALTUS}

La comparaison des données altimétriques issues du maximum du seuil 1 (75\%) et des mesures manuelles a donc démontré la capacité des ALTUS à réaliser une mesure fiable du niveau sédimentaire malgré la présence de feuilles de zostères (voir tableau 1 et figure 3). Cependant, malgré la coupe des zostères sous les capteurs, il apparaît que pour certaines périodes (du 30/05 au 24/08, et du 20/10 au 25/11, respectivement pour la station d'herbier dense et pour la station d'herbier moyennement dense), les mesures correspondant au niveau sédimentaire sont bruitées par les feuilles de zostères (voir figure $3 \mathrm{a}$ et $3 \mathrm{~b}$ ). Aussi, bien que les coupes ne s'avèrent pas nécessaires pour obtenir une mesure précise du niveau du substrat lorsque les zostères sont de petite taille et ont un faible I.S.F., lorsque les herbiers atteignent un certain niveau de développement (I.S.F.>5 $\mathrm{m}^{2} / \mathrm{m}^{2}$ ), les coupes semblent nécessaires pour garantir la qualité du signal sédimentaire.

La figure 4 représente, pour les différentes stations, les distances maximales entre le point de mesure et le transducteur, calculées par salve pour le seuil 1 (75\%, points noirs) et les distances minimales calculées pour le seuil 4 (10\%, croix grises). Sur le même graphique, ont été ajoutées les données mensuelles de longueur moyenne de feuilles (voir figure 4, pointillés noirs). Celles-ci ont été corrigées avec la donnée ALTUS du maximum du seuil 1 afin de tenir compte des évolutions altimétriques du substrat sédimentaire au cours du temps. Les données du seuil 4 (voir figure 4, croix grises) présentent une distribution plus dispersée que celles du seuil 1 (points noirs). La base du nuage de points du seuil 4 coïncide avec le nuage de points du seuil 1 , pour toutes les stations d'herbier (voir figure 4a à 4c), tandis que pour la station d'estran nu, les nuages de points des seuils 1 et 4 sont confondus (voir figure $4 d$ ). De plus, on peut remarquer que les mesures de l'élévation pour le seuil 4, correspondent plutôt bien aux longueurs moyennes de feuilles mesurées par prélèvements (voir figures 4a à 4c), principalement pour la station d'herbier peu dense, pour laquelle une seule coupe a été effectuée au début de la période de mesure (voir figure 4c). Il apparaît également qu'à la suite d'une coupe, l'élévation pour le seuil 4 est plus faible, puis augmente progressivement. Ce qui est interprétée comme la repousse des zostères consécutive à la coupe. Ensuite, à la fin 
de la période de mesure, pour les stations d'herbier dense (voir figure 4a) et d'herbier moyennement dense (voir figure $4 \mathrm{~b}$ ), le nuage de points est tronqué à une valeur constante. Ceci s'explique par le fait que les ALTUS ne fournissent pas de données pour une distance entre le transducteur et le point de mesure inférieure à $100 \mathrm{~mm}$, et par le remplacement des deux ALTUS par de nouveaux, positionnés plus près du sédiment.

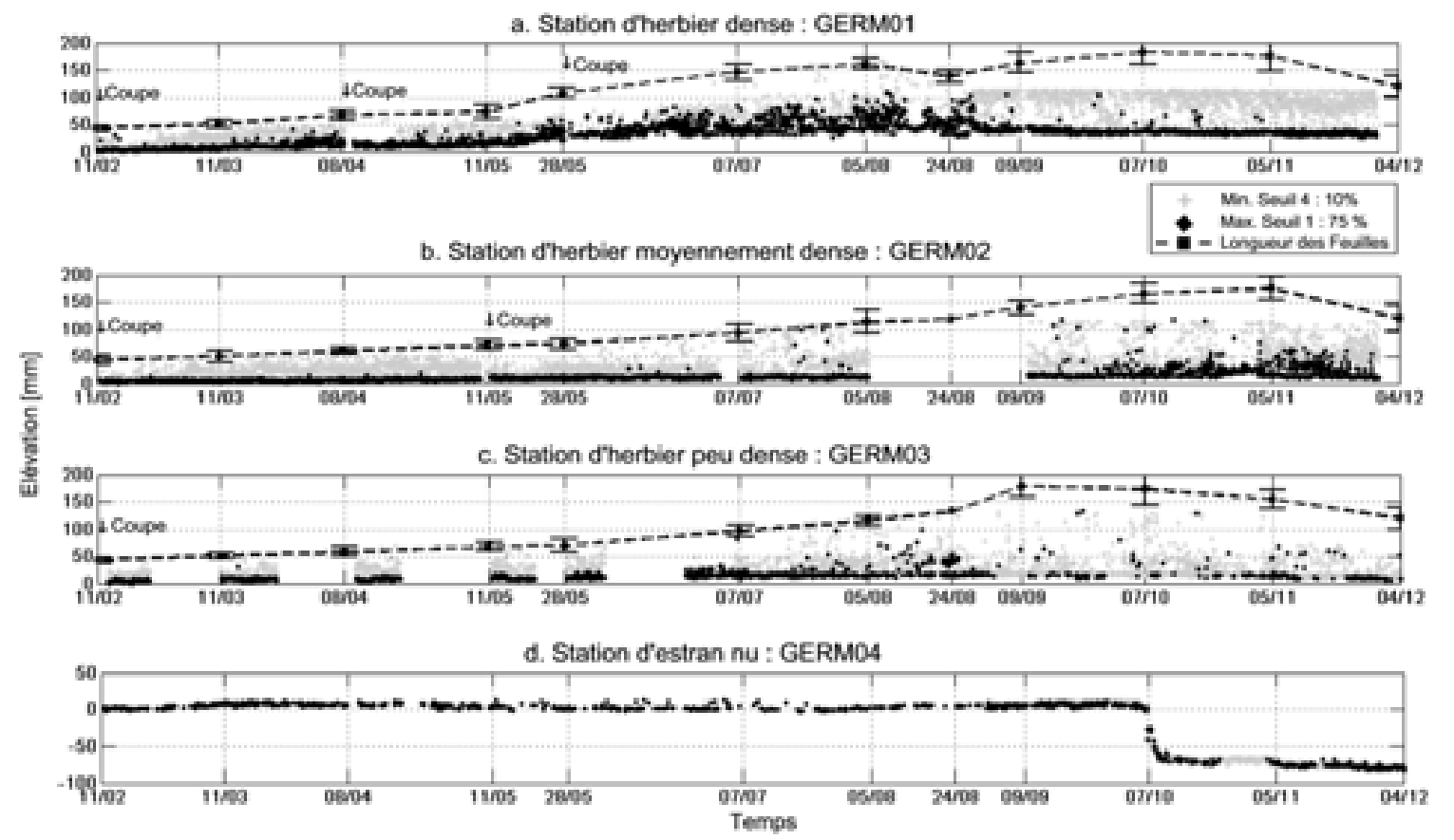

Figure 4. Mesures ALTUS (seuil 1-points noirs, et seuil 4-croix grises) et données de longueurs de feuille moyennes (pointillés noirs). Les quatre stations sont représentées : (a.) l'herbier dense, (b.) l'herbier moyennement dense, (c.) l'herbier peu dense et (d.)

\section{l'estran $n u$.}

La confrontation entre les données altimétriques du seuil 4 minimisé et les données de longueur de feuilles issues des prélèvements biométriques a mis en évidence des tendances similaires, principalement durant les premiers mois de mesures. Ceci permettant de s'interroger sur la capacité des ALTUS à constituer un moyen d'estimer de la taille des feuilles. Cependant, la mesure ALTUS qui nécessite une densité de réflecteurs (feuilles) suffisante pour atteindre le niveau d'énergie du seuil 4, sous-estime la longueur des feuilles durant la période estivale. Cette sous-estimation pourrait être induite par les coupes sous les capteurs qui engendreraient un retard dans la croissance des feuilles, par rapport aux prélèvements, pour lesquels, les zostères n'ont jamais subi de coupe. Une seconde explication, complémentaire, serait l'effet du courant sur la courbure des feuilles. En effet, la mesure de la longueur des feuilles par la biométrie, 


\section{XI $I^{\text {èmes }}$ Journées Nationales Génie Côtier - Génie Civil}

Les Sables d'Olonne, 22-25 juin 2010

donne la longueur absolue des feuilles, alors que la mesure ALTUS donne une mesure relative, les herbiers étant immergés et soumis aux courants de marée, donc courbés.

Les longueurs de feuilles (par soustraction entre le seuil 4 le seuil 1) mesurées par les ALTUS ont été représentées dans la figure 5 pour quelques cycles de marée sans vent, et comparées à la hauteur d'eau enregistrée. On observe, au début du flot, que les longueurs mesurées augmentent progressivement jusqu'à l'étale de pleine mer, puis diminuent durant le jusant. Ainsi, les hauteurs de l'herbier mesurées par les ALTUS peuvent être mises en relation avec les courants de marée. Cette relation permet de valider le rôle du courant sur la répartition verticale et la hauteur effective de la canopée (ABDELRHMAN, 2007). La capacité de mesure des longueurs de feuille par les ALTUS en conditions réelles pourrait avoir un intérêt certain pour la modélisation. En effet, de nombreuses formulations mathématiques utilisées dans les modèles numériques pour simuler l'écoulement en présence de végétation ont recours à ce paramètre, qui devait jusqu'alors être calculé empiriquement à partir d'expérimentations in-situ ou en canal à courant (ABDELRHMAN, 2003, 2007).

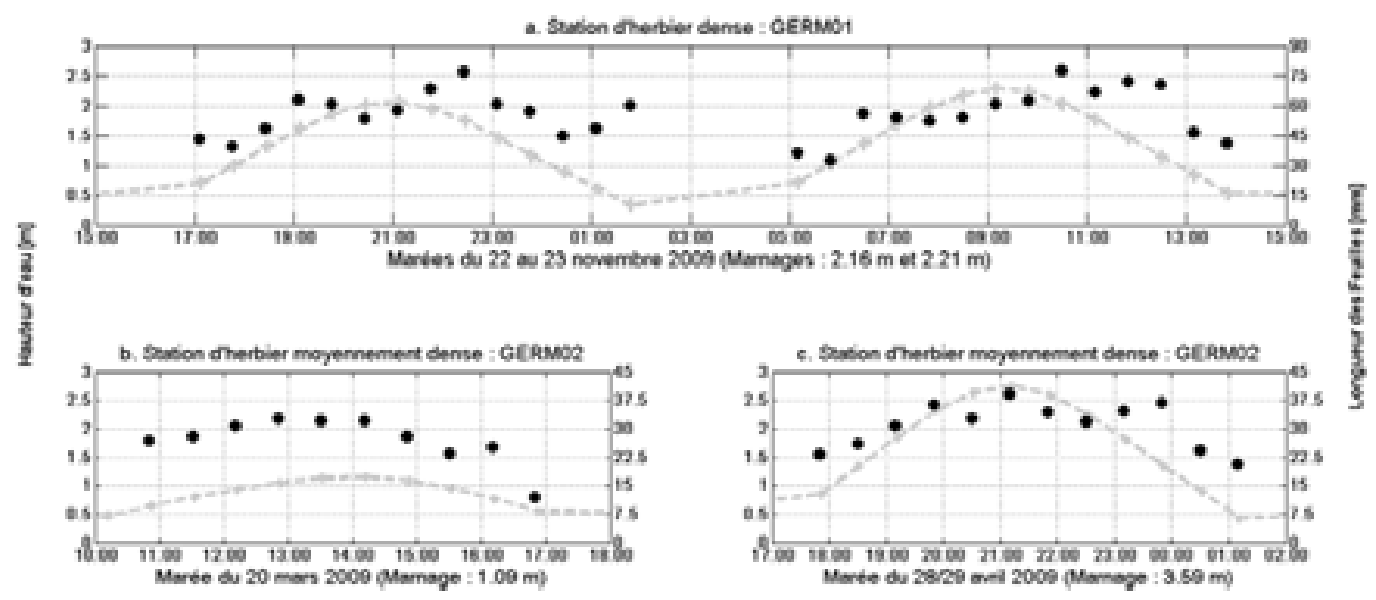

Figure 5. Comparaison des hauteurs d'eau (pointillés gris) et des longueurs de feuille mesurées par les ALTUS (Seuil4-Seuill, cercles noirs) pour des cycles de marée en conditions de clapot nul. (a) marées moyennes en novembre pour la station d'herbier dense, (b et c) marées de morte-eau et de vive-eau en mars et octobre respectivement, pour la station d'herbier moyennement dense.

\subsection{Les herbiers de zostères : rôle de pièges à sédiments et protecteur contre l'érosion}

L'exploitation des données altimétriques a également montré une tendance marquée à l'accrétion sédimentaire durant la période de croissance de l'herbier (voir figure 3), traduisant la mise en place continue d'un dépôt de sédiments frais durant le printemps et le début de l'été. L'épaisseur du dépôt mis en place durant la période de croissance des zostères (respectivement 40, 13 et $15 \mathrm{~mm}$ pour les stations d'herbier dense, moyennement dense et peu dense), s'avère très supérieure aux résultats obtenus pour 
l'espèce Zostera marina en Mer de Wadden (BOS et al., 2007) pour laquelle l'accrétion n'excédait pas 5 à $7 \mathrm{~mm}$. Ainsi, le rôle joué par les herbiers de zostères naines pour le piégeage des sédiments semble important, comparé à l'évolution observée pour la station témoin d'estran nu, pour laquelle le bilan sédimentaire entre février et la mijuillet est nul.

Ensuite, de la mi-juillet au 4 décembre, les niveaux sédimentaires se stabilisent puis diminuent faiblement (diminution $<10 \mathrm{~mm}$ ) pour les stations colonisées, pouvant traduire un arrêt du piégeage des sédiments associé au tassement du dépôt frais, ou à une phase d'érosion. Ce comportement diffère de celui observé sur la station d'estran nu, où une phase d'érosion continue est mesurée (-25 mm entre le 10/10 et le 4/12), corrélée avec l'augmentation de la fréquence et de l'intensité des tempêtes automnales. Il est à noter cependant, que la phase majeure d'érosion (-60 mm en $48 \mathrm{~h})$ du 7/10 a été causée par la déstabilisation du sédiment autour de l'ALTUS lors des visites mensuelles et, n'est donc pas intégrée dans le calcul du bilan sédimentaire pour cette station. Ainsi, le bilan sédimentaire des stations situées dans l'herbier reste toutefois positif sur la totalité de la période d'enregistrement (respectivement $+24 \mathrm{~mm},+10 \mathrm{~mm}$ et $+8 \mathrm{~mm}$ pour les stations d'herbier dense, moyennement dense et peu dense), alors que pour la station de vase nue, le bilan sédimentaire serait de $-25 \mathrm{~mm}$.

Le rôle joué par les zostères naines dans la dynamique sédimentaire semble important et se traduit par un fort pouvoir de piégeage durant la période de croissance des zostères. L'absence d'érosion pour les stations colonisées sur les 10 premiers mois du suivi pourrait se traduire à long terme par un exhaussement continu des estrans colonisés. Il est toutefois nécessaire de connaître la dynamique sédimentaire en période hivernale (décembre-janvier) pour pouvoir conclure sur cette question.

Dans ce contexte, la régression de la surface des herbiers de zostères du bassin d'Arcachon pourrait avoir un effet significatif sur la redistribution des stocks sédimentaires. En effet, on peut imaginer qu'en l'absence d'herbiers, le piégeage des matières en suspension serait faible durant les périodes calmes, et les sédiments pourraient être remis en suspension durant les tempêtes, sans avoir eu le temps de se consolider. Les sédiments ainsi remobilisés pourraient alors se déposer dans les chenaux possédant une faible énergie, principalement dans les parties internes du bassin, où le transport résiduel est également contrôlé par le flot (PLUS et al., 2006). De plus, le suivi cartographique des herbiers de zostères naines du Bassin d'Arcachon (DALLOYAU et al., 2009) montre que la régression de surface intervient principalement dans les parties les plus internes du bassin. Le SIBA, organisme chargé de l'entretien des voies navigables dans le Bassin d'Arcachon a observé depuis quelques années, conjointement à la régression des herbiers dans les parties orientales du bassin, la nécessité croissante de draguer les chenaux peu profonds de l'Est du bassin (SIBA, comm. pers.). 


\section{Conclusion}

Cette étude a permis de mettre en évidence la capacité des altimètres ALTUS à fournir une mesure fiable du niveau sédimentaire en présence d'herbiers. La possibilité d'utiliser des ALTUS afin d'obtenir une estimation de la hauteur effective de l'herbier soumis aux forçages hydrodynamiques, paramètre important pour la modélisation numérique de l'hydrodynamique en présence d'herbier a également été avancée. Cependant, des mesures complémentaires sont nécessaires afin de vérifier l'hypothèse selon laquelle, la sous-estimation des longueurs de feuilles serait en partie induite par la coupe de l'herbier sous les capteurs. Pour cela, une comparaison sur une même station des données d'un ALTUS sous lequel les feuilles sont coupées à intervalles réguliers avec les données d'un ALTUS sous lequel les feuilles ne seront jamais coupées devrait être réalisée.

Ensuite, le rôle important de piégeage des sédiments par les herbiers, durant la période de croissance printanière et estivale a été démontré, ainsi que le rôle protecteur des herbiers contre l'érosion malgré la dégénérescence automnale du feuillage et l'augmentation de la fréquence et de l'intensité des coups de vent générant du clapot. Néanmoins, l'influence des paramètres biométriques, tels que la densité de pousses, sur la capacité de piégeage et de protection contre l'érosion doit être étudiée plus en détail, de même que la représentativité des tendances saisonnières obtenues pour le site atelier avec d'autres sites répartis dans le bassin d'Arcachon. Enfin, la modélisation numérique devrait permettre d'appréhender les conséquences sur les bilans sédimentaires de la lagune, de la régression des herbiers intertidaux de Zostera noltii sur le long terme.

Remerciements : Les auteurs remercient le SIBA (Syndicat Intercommunal du Bassin d'Arcachon) et l'Ifremer pour le financement de la thèse dans laquelle cette étude s'inscrit, ainsi que pour les moyens instrumentaux mis à disposition. Sont également remerciés : les marins du bateau PLANULA IV, de l'Institut National des Sciences de l'Univers, Isabelle Auby de l'Ifremer d'Arcachon pour son aide à la mise en place du protocole de prélèvement et de traitement biométrique, ainsi que toutes les personnes de l'UMR EPOC, techniciens, ingénieurs et chercheurs ayant apportés leur aide lors des missions de terrain.

\section{Références bibliographique}

ABDELRHMAN M.A. (2003). Effect of eelgrass Zostera marina canopies on flow and transport. Marine Ecology Process Series, ${ }^{\circ} 248$, pp 67-83.

ABDELRHMAN M.A. (2007). Modeling coupling between eelgrass Zostera marina and water flow. Marine Ecology Progress Series, $\mathrm{n}^{\circ}$ 338, pp 81-96. doi:10.3354/meps338081 AMOS C.L., BERGAMASCO A., UMGIESSER G., CAPPUCCI S., CLOUTIER D., DENAT L., FLINDT M., BONARDI M., CRISTANTE S. (2004). The stability of tidal 
Thème 2 - Dynamique sédimentaire et transports des particules

flats in Venice Lagoon - the results of in-situ measurements using two benthic, annular flumes. Journal of Marine Systems, $\mathrm{n}^{\circ}$ 51, pp 211-241. doi:10.1016/j.jmarsys.2004.05.013 BASSOULLET P., LE HIR P., GOULEAU D., ROBERT S. (2000). Sediment transport over an intertidal mudflat : field investigation and estimation of fluxes within the "Baie de Marennes-Oléron" (France). Continental Shelf Research, ${ }^{\circ}$ 20, pp 1635-1653. doi:10.1016/S0278-4343(00)00041-8

BOS A.R., BOUMA T.J., DE KORT G.L.J., VAN KATWIJK M.M. (2007). Ecosystem engineering by annual intertidal seagrass beds : Sediment accretion and modification. Estuarine, Coastal and Shelf Science, $\mathrm{n}^{\circ}$ 74, pp 344-348. doi:10.1016/j.ecss.2007.04.006 DALlOYAU S., TRUT G., PLUS M., AUBY I. (2009). Caractérisation de la qualité biologique des Masses d'Eau Côtières : Cartographie des herbiers de Zostera noltii et Zostera marina du Bassin d'Arcachon. Rapport Ifremer RST /LER/AR/09-003: 51.

DE BOER W.F. (2007). Seagrass-sediment interactions, positive feedbacks and critical thresholds for occurrence : a review. Hydrobiologia, $\mathrm{n}^{\circ}$ 591, pp 5-24. doi:10.1007/s10750-007-0780-9

DELOFFRE J., VERNEY R., LAFITE R., LESUEUR P., LESOURD S., CUNDY A.B. (2007). Sedimentation on intertidal mudflats in the lower part of macrotidal estuaries : Sedimentation rhythms and their preservation. Marine Geology, $\mathrm{n}^{\circ} 241$, pp 19-32. doi:10.1016/j.margeo.2007.02.011

FONSECA M.S., FISHER J.S. (1986). A comparison of canopy friction and sediment movement between four species of seagrass with reference to their ecology and restoration. Marine Ecology Process Series, $\mathrm{n}^{\circ}$ 29, pp 15-22.

GACIA E., DUARTE C.M., MARBA N., TERRADOS J., KENNEDY H., FORTES M.D., TRI N.H. (2003). Sediment deposition and production in SE-Asia seagrass meadows. Estuarine, Coastal and Shelf Science, $\mathrm{n}^{\circ}$ 56, pp 909-919. doi:10.1016/S0272-7714(02)00286-X

JESTIN H., BASSOULET P., LE HIR P., L'YAVANC J., DEGRES Y. (1998). Development of ALTUS, a high frequency acoustic submersible recording altimeter to accurately monitor bed elevation and quantify deposition or erosion of sediments. Ocean Conference Record (IEEE), n 1, pp 189-194.

MOLLER I. (2006). Quantifying saltmarsh vegetation and its effects on wave height dissipation : Results from a UK East coast saltmarsh. Estuarine, Coastal and Shelf Science $\mathrm{n}^{\circ}$ 69, pp 337-351. doi:10.1016/j.ecss.2006.05.003

PLUS M., MAURER D., STANISIERE J.-Y., DUMAS F. (2006). Caractérisation des composantes hydrodynamiques d'une lagune mésotidale : le Bassin d'Arcachon. Rapport IFREMER, RST/LER/AR/06-007 : 52 p + Annexes.

THOMPSON C.E.L., AMOS C.L., UMGIESSER G. (2004). A comparison between fluid shear stress reduction by halophytic plants in Venice Lagoon, Italy and Rustico Bay, Canada--analyses of in situ measurements. Journal of Marine Systems 51, pp 293-308. doi:10.1016/j.jmarsys.2004.05.017 